\title{
Discussion about Replacing Ferrite Inductance with Amorphous Inductance in Switch Circuit
}

\author{
ZHANG $\mathrm{Yi}^{1, \text { a }}$ \\ ${ }^{1}$ Jiangxi Vocational Technical College of Industry Trade, Nanchang 330038, China \\ azhangyijx@163.com
}

Keywords: frequency switching power supply technology; switch circuit; amorphous inductance; ferrite inductance; research;

\begin{abstract}
Along with the development of high frequency switching power supply technology, people on the electromagnetic compatibility, noise pollution more and more seriously, the anti EMI technology has become a new technology and new industry, solves the problems of electromagnetic compatibility of electronic equipment is becoming more and more urgent, all kinds of anti electromagnetic interference suppression of noise and other magnetic devices widely used, one of the key elements of these devices is the inductance device. Switching power supply with its advantages of high efficiency, small volume, output stability good and rapid development. However, due to the high frequency, high $\mathrm{di} / \mathrm{dt}$ and high $\mathrm{dv} / \mathrm{dt}$ makes the problem of electromagnetic interference of switching power supply in the process of the work is very outstanding. China has been in a new 3C authentication to replace the CCIB and CCEE certification, which makes the switching power supply for the electromagnetic compatibility requirements more detailed and strict. Now, how to reduce or even eliminate the problem of EMI switching power supply has become a global switch power supply designers and electromagnetic compatibility (EMC) designers are very concerned problems.
\end{abstract}

\section{Introduction}

The inductance design of small, medium and frequency power with ferrite core $(10 \sim 300 \mathrm{kHz})$ [3]become more and more popular. However, due to the nonlinear ferrite materials, allowing the calculation of inductance is complicated, the existing amorphous inductive ferrite replacement circuit in the switch circuit position, so this to these two kinds of inductance compared to measure these two kinds of inductance in the application of the switching circuit about the advantages and disadvantages.

\section{The concept and characteristics of ferrite inductor}

Ferrite inductor is a special form of inductance. Its basic construction is in the ferrite magnetic column through a wire, early also called it a ferrite bead. We put the ferrite inductance called inductance, that is to say it has the general characteristic similar inductance, but also has the special characteristics of its own. Form depends on the special characteristics of ferrite inductor with ferrite core material. Ferrite is a magnetic, which is composed of a magnetic powder sintering and combination of different characteristics.

Ferrite material is magnesium alloy of iron or iron nickel alloy, the material has high permeability, can he is between the coil winding inductance at high frequency and high resistivity capacitor produced under the condition of minimum. Ferrite materials are usually used in high frequency case, because they mainly in the low frequency range of inductance characteristic make the line loss is very small. [4]In the case of high frequency, they are mainly reactance characteristic and change with frequency. 


\section{Mechanism of ferrite inductor}

The working mechanism of the working mechanism of ferrite inductance and ordinary inductance has the very big difference. Ordinary inductance has several characteristics: one is the current in the inductor to hysteresis voltage, the two is the inductance will produce different inductance on the AC signal [3] is different, three is the inductive energy storage function. These characteristics in the ferrite inductance in some no longer exist, although some exist but the mechanism is different. Inhibitory effect of ordinary inductance on the AC signal is generated on the AC signal using inductive reactance. Ferrite inductance is different, when the composition of ferrite inductance material of $\mathrm{Mn} \mathrm{Zn}$ ferrite in low frequency with high permeability about can do more than a few hundred to 2000, but at higher frequencies the great loss of its magnetic. But the nickel zinc ferrite although initial permeability is low, but its high frequency magnetic loss is small, can work at higher frequency. People use Mn Zn ferrite in high frequency magnetic loss larger properties, it is made into a cylinder and the center through a wire, and it made the ferrite inductance.

\section{Inhibiting the role switching noise of the saturable inductor}

Switching power supply usually work in tens of hertz to several hundred $\mathrm{Hz}$ frequency range, flowing through the high reverse recovery current will power the secondary side rectifier diode in high frequency shutdown process, so the formation of the power supply conducting the spike noise is an important part of switch power supply noise. Try to reduce the rectifier diode reverse recovery current, thereby reducing the conduction and radiation noise of switch power supply switch is an important aspect in the design of power supply.(showed in Fig 1) [1]

\begin{tabular}{|l|l|}
\hline $\mathrm{I} / \mathrm{A}$ & $\mathrm{L} / \mathrm{mH}$ \\
\hline 1 & $8-23$ \\
\hline 3 & $2-4$ \\
\hline 6 & $0.4-0.8$ \\
\hline 10 & $0.2-0.3$ \\
\hline 12 & $0.1-0.15$ \\
\hline 15 & $0.0-0.08$ \\
\hline
\end{tabular}

Fig 1 The relationship between the inductance range and rated current

Magnetic amplifier regulator is using saturable inductor guide physical characteristics on delay to control the switch duty ratio of the power supply and power output, a method for stabilizing output voltage of power supply. In a saturable inductor with proper sampling and control components, adjusting the turn-on delay time, can constitute the most common magnetic amplifier regulator circuit. Normally, it is an input $110 \mathrm{Vac} 220 \mathrm{Vac} / 50 \mathrm{HZ}$, output 5Vdc/20A, 12Vdc/10A [2] double forward switching power supply, in which $5 \mathrm{~V}$ is the main control circuit. The auxiliary road $12 \mathrm{~V}$ output current is large, the precision of voltage and load stability have higher requirements, using ordinary methods of voltage stabilizing effect is not very ideal, if the use of magnetic amplifier regulator technology can solve this problem satisfactorily, the voltage stabilizing circuit of simple structure. 110Vac 220Vac/50HZ input through the active power factor correction circuit to enhance the voltage, DC voltage output $400 \mathrm{Vdc}$ with power modules in the primary and secondary windings N1 peak output for the 20V. Duty ratio is about 25\% of the square wave voltage; the peak output voltage of secondary winding of $\mathrm{N} 2$ is $50 \mathrm{~V}$. [2]

The following analysis of the working principle of magnetic amplifier and voltage stabilizing circuit, a voltage stabilizing process curve can be magnetic induction intensity changes saturated inductance from the above analysis, due to the insertion of saturable inductance Ls, makes the auxiliary road conduction time T 'ON. [4] a' is the duty ratio of the main loop is small, based on the duty cycle adjustment a 'to finally realize the purpose of auxiliary circuit output voltage regulator. a' has a large range, in the auxiliary road completely no-load, a' almost can be adjusted to 0 , so that 
the secondary road with a load stability and high regulation accuracy. The duty ratio a 'according to the following formula:

\section{$\mathrm{T}$ 'a' $=$ ON/T $=($ TON-DT $) / \mathrm{T}$}

Type: TON: the main circuit conduction time; T: switch cycle power supply; DT: Ls turn-on delay time. [4]

\section{Analysis of interference source switching power supply}

The most fundamental reason of electromagnetic interference of switching power supply is generated, the generated in the working process of high $\mathrm{di} / \mathrm{dt}$ and high $\mathrm{dv} / \mathrm{dt}$, [5] which produces the surge current and voltage spike formation of interference source. Reverse large capacitance frequency rectifier filtering using the charge discharge, voltage switching, and output rectifier diode tube high-frequency work when the recovery current is this kind of interference source. For the rectangular wave, the reciprocal of the period determines the fundamental frequency wave; time and down time of up to two times the reciprocal of pulse edge determines the frequency components caused by the edge of the frequency value, typically in the range of $\mathrm{MHz}$, and its harmonic frequency is higher. These high frequency signals turn to the switching power supply basic signal, especially the interference signal control circuit (showed in Fig 2). [5]

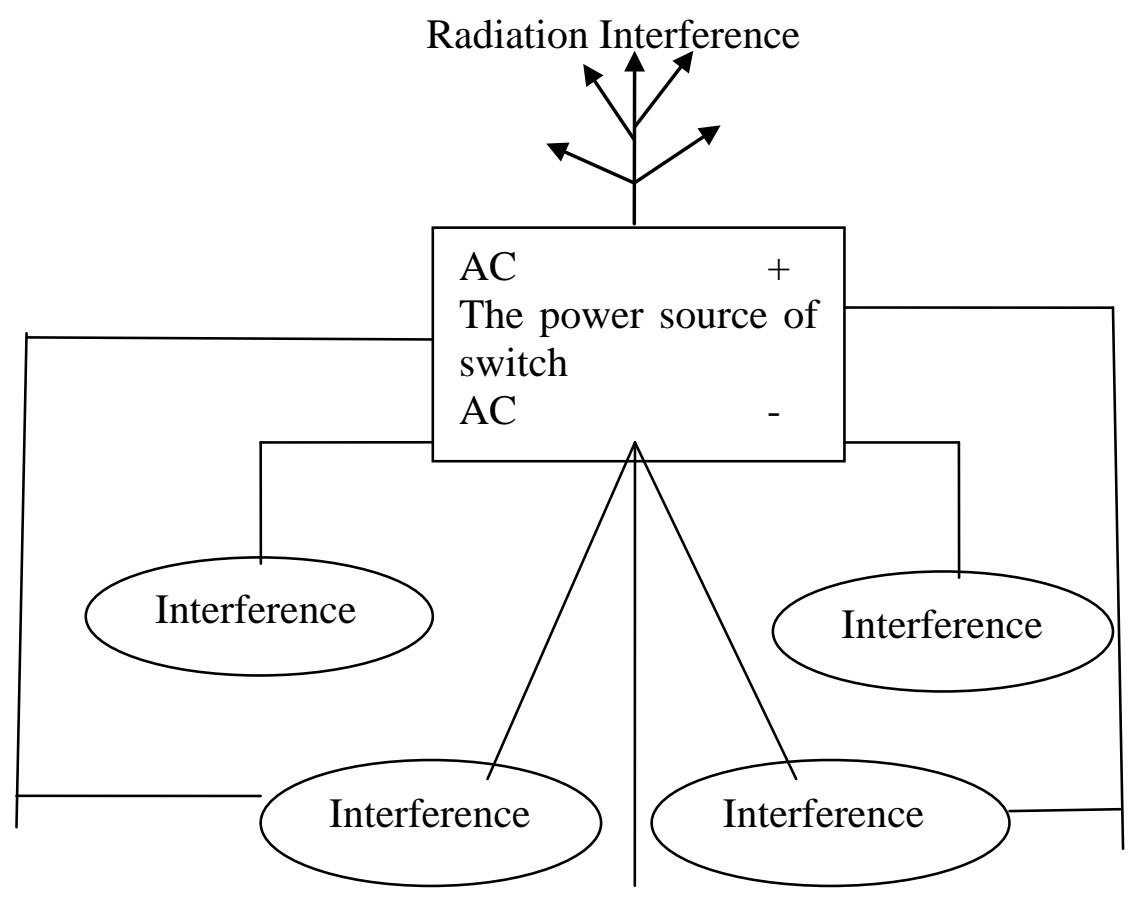

Fig 2 the interference signal control circuit

\section{Conclusion}

Switching power supply with its advantages of high efficiency, small volume, output stability good and rapid development. However, due to the high frequency, high di/dt and high dv/dt makes the problem of electromagnetic interference of switching power supply in the process of the work is very outstanding. China has been in a new 3C authentication to replace the CCIB and CCEE certification, which makes the switching power supply for the electromagnetic compatibility requirements more detailed and strict. 


\section{References}

[1] FenG yun, Electronic Current Transformer [J], Science and Technology, 2011, (08):49.

[2] Gu Yunfei, Practical Applications of Electronic Current Transformer [J]. Journal of Architecture, Institute of Command and Technology of Equipment, 2011: 04.

[3] Han Xiuzhi. The Security Strategy [J], Cooperation of Economy and Technology, Cloud Computing Platform Based on 2011, (08): 125 - 127.

[4] Lu Jianwei. The Analysis and Solution of Electronic Current Transformer Scheduling [J]. Science and Technology Information, 2011:15.

[5] Wang zhaomei, The Application of Electronic Current Transformer [J], Science and Technology Information, Influence on Higher Education of Cloud Computing, 2010, (10): 49-61. 